\title{
PRIMARY CENTRAL NERVOUS SYSTEM LYMPHOMAS
}

\author{
Adriana Octaviana Dulamea \\ Department of Neurology, Fundeni Clinical Institute, \\ „Carol Davila“ University of Medicine and Pharmacy, Bucharest
}

\begin{abstract}
Primary central nervous system lymphoma (PCNSL) is an aggressive non-Hodgkin B-cell lymphoma with characteristic clinical behavior, biological features and poor prognosis despite complex treatment. PCNSL has a median survival of 17 to 45 months in immunocompetent patients, and only $20-30 \%$ of cases can be cured successfully. Clinical outcome has improved since the advances in combination chemotherapy protocols, addition of whole brain radiation therapy, encouraging responses of rituximab adminsitration in refractory PCNSL and autologous hematopoietic stem-cell transplantation as consolidative therapy. The author review the recent data on pathogenesis, diagnostic methods and treatment strategies of PCNSL in immunocompetent patients.
\end{abstract}

Keywords: primary central nervous system lymphoma, pathogenesis, magnetic resonance imaging, 18-fluorodeoxyglucose positron emission tomography

\section{INTRODUCTION}

Primary central nervous system lymphomas (PCNSL) are rare tumors of the central nervous system (CNS), representing approximately $2 \%$ of all primary CNS tumors and less than $1 \%$ of nonHodgkin lymphomas. (1) The concept and understanding of central nervous system (CNS) lymphoma have greatly evolved in the past few years and it is reflected in the complex WHO Classification of Tumors of Hematopoietic and Lymphoid Tissue. Primary central nervous system lymphoma is now restricted to primary diffuse large B-cell lymphoma confined to the CNS (brain, spinal cord, eyes, meninges, and cranial nerves) (2-4). Many other lymphoma subtypes, some of which are primary or exclusive to the CNS, such as lymphomas of the dura and immunodeficiency-associated lymphomas, are excluded from this definition. Intravascular large B-cell lymphoma (IVLBCL), also a rare form of disseminated B-cell lymphoma characterized by selective growth of lymphoma cells within small vessels/capillary lumina, can also present clinically with a predominant neurologic variant secondary to
CNS involvement (4) The majority of PCNSL are sporadic and the incidence increases with age. A minority are attributable to immunosuppressed states, including HIV infection or iatrogenic immunosuppression following organ transplantation. (5) PCNSL in immunocompetent patients are not associated with Epstein-Barr virus and affect older populations, with a median age of 55 years, and with a slight male predominance (male-to-female ratio $3: 2$ ). Higher age has consistently been identified as a negative prognostic factor in patients with PCNSL, the poor outcome being attributed to less intensive treatment administered and also to a fundamental difference in the response to treatment.

\section{PATHOGENESIS}

The pathogenesis of PCNSL remains largely unclear, which is partly due to the rarity of the tumor tissue available for research studies. Transcriptomic studies have identified deregulated genes involved in the IL4/JAK/STAT6, cell adhesion-related, unfolded protein response (UPR) and apoptosis signaling pathways (6-9). Copy number variation 
studies $(8,10-12)$ have revealed frequent chromosome losses affecting the 6q, 6p21.32 and 9p21 regions. However, the mutational landscape of PCNSL is still poorly known. A whole exome sequencing strategy has successfully identified pivotal gene mutations in several hematologic and brain malignancies $(13,14)$ Eight genes of interest have been further investigated by focused resequencing in a large cohort. The study revealed high mutation rates for genes already described as mutated in PCNSL such as MYD88 (38\%), CD79B $(30 \%)$, PIM1 (22\%) and TBL1XR1 (19\%) and for genes not previously reported to be involved in PCNSL tumorigenesis such as ETV6 (16\%), IRF4 (14\%), IRF2BP2 (11\%) and EBF1 (11\%). PCNSL demonstrates genetic heterogeneity and mutational pattern similarities with extracerebral diffuse large $\mathrm{B}$ cell lymphomas, particularly of activated B-cell (ABC) type, suggesting shared underlying biological mechanism. (15) There are several hypotheses which aim to explain the pathogenesis of PCNSL. Since the central nervous system lacks lymphoid tissue or lymphatic vessels, PCNSL may be caused by the monoclonal proliferation of continuously trafficking T-cells or B-cells in CNS, or the specific tropism of neoplastic T or B lymphocytes for CNS where they escape from the attack of the intact peripheral immune system (16). Malignant transformation of $\mathrm{T}$ or $\mathrm{B}$ cells after a benign inflammatory process within the CNS may also be the origin of PCNSL (17). Additionally, neoplastic lymphocytes that are normally eradicated by the peripheral immune system may escape to the CNS. The special environment, so-called "sanctuary site", where is free from attack of the immune system and penetration of chemotherapeutic agents by blood-brain barrier is deeply related to malignant transformation. (18) This has important therapeutic implications. At presentation and especially at recurrence, there is a heavy disease burden that exists behind an relatively intact blood-brain barrier. These individual tumor cells invade brain and receive their nutrition from normal cerebral blood vessels. They are not supported by the permeable tumor neovessels that sustain bulky tumor. Therefore, effective treatment of PCNSL requires agents that penetrate the blood-brain barrier to reach these individual cells. These data also suggest that antiangiogenic agents are unlikely to be effective against this microscopic disease.

\section{CLINICAL FEATURES}

Clinical presentation of PCNSL is frequently characterized by focal mass lesions signs in more than $50 \%$ of cases. In 248 immunocompetent patients, $43 \%$ had neuropsychiatric signs, $33 \%$ had increased intracranial pressure, $14 \%$ had seizures, and $4 \%$ had ocular symptoms at the time of presentation. (19) Seizures are less common than with other types of brain tumors, probably because PCNSL involves predominantly subcortical white matter rather than epileptogenic gray matter. Patients rarely present with $\mathrm{B}$ symptoms such as fever, weight loss, or night sweats that are commonly associated with other forms of NHL (20).

\section{DIAGNOSTIC EVALUATION}

The International PCNSL Collaborative Group has established guidelines for the diagnostic evaluation of a patient with suspected PCNSL (Table 1). (21) The guidelines express the goal to evaluate the baseline extent of disease in order to confirm that the disease is restricted to CNS. Physical examination should include palpation for enlarged lymph nodes as well as testicular examination in males be-

TABLE 1. International PCNSL Collaborative Group Guidelines for Baseline Evaluation for Clinical Trialsa

\begin{tabular}{|l|l|l|l|}
\hline Pathology & Clinical & Laboratory & Imaging \\
\hline $\begin{array}{l}\text { Centralized review of } \\
\text { pathological findings }\end{array}$ & $\begin{array}{l}\text { Complete medical and } \\
\text { neurological examination }\end{array}$ & HIV serology & $\begin{array}{l}\text { Contrased enhanced cranial } \\
\text { MRI }^{\text {b }}\end{array}$ \\
\hline Immunophenotyping & $\begin{array}{l}\text { Dilated eye examination, } \\
\text { including slitlamp evaluation }\end{array}$ & Serum LDH level & $\begin{array}{l}\text { CT of chest, abdomen and } \\
\text { pelvis }\end{array}$ \\
\cline { 2 - 4 } & $\begin{array}{l}\text { Recording of prognostic } \\
\text { factors (age, performance status) }\end{array}$ & $\begin{array}{l}\text { CSF cytiology, flow cytometry, } \\
\text { immunoglobulin heavu-chain PCR24 h } \\
\text { urine collection for creatinine clearence }\end{array}$ & $\begin{array}{l}\text { Bone marrow biopsy with } \\
\text { aspirate }\end{array}$ \\
\cline { 2 - 5 } & $\begin{array}{l}\text { Serial evaluation of cognitive } \\
\text { function }^{c}\end{array}$ & $\begin{array}{l}\text { Testicular ultrasonography in } \\
\text { elderly men }\end{array}$ \\
\hline
\end{tabular}

Abbreviations: CSF, cerebrospinal fluid; CT, computed tomography; HIV, human immunodeficiency virus; LDH, lactate dehydrogenase; MRI, magnetic resonance imaging; PCNSL, primary central nervous system lymphoma; PCR, polymerase chain reaction.

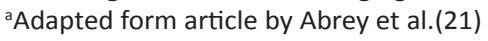

${ }^{b}$ Contrast enhanced cranial CT should be obtained in patients who have a contraindication for MRI or who cannot tolerate MRI

'Mini-Mental State Examination is used commonly, although improvement instruments are developed.

${ }^{\mathrm{d}}$ For patients who will receive high-dose methotrexate. 
cause testicular lymphoma has a predilection to disseminate to the brain parenchyma. Contrast enhanced cranial magnetic resonance imaging (MRI), postcontrast cranial computed tomography if MRI is contraindicated, lumbar puncture if not contraindicated (for cell count, protein and glucose measurement, cytology, immunoglobulin heavy-chain gene rearrangement studies, and flow cytometry studies), ophthalmologic examination including slitlamp evaluation, computed tomography of the chest, abdomen, and pelvis, and bone marrow biopsy should be performed. Blood tests for HIV, complete blood cell count, basic metabolic profile, and lactate dehydrogenase level are also recommended. Testicular ultrasonography should be considered in men.

\section{Neuroimaging}

\section{Magnetic resonance imaging}

On MRI, PCNSL should be differentiate from CNS involvement of a systemic nonHodgkin lymphoma, from glioma and metastases. Although primary CNS presentation of systemic NHL is very rare, staging of all patients first diagnosed as having lymphoma in the CNS is mandatory to exclude this possibility. Neuroimaging findings differ in immunocompetent from immunodeficient patients. Although some imaging findings are characteristic of PCNSL, the frequency of atypical findings on conventional neuroimaging is increasing. Atypical neuroimaging findings do not rule out PCNSL, even in immunocompetent patients (22).

The typical magnetic resonance imaging (MRI) lesions of PCNSL (23-25) include single or multiple intra-axial, homogenous, contrast enhancing lesions with marked perilesional edema and restricted diffusion, usually contacting the cerebrospinal fluid surface. Lesions not in contact with the subarachnoid space are low-grade PCNSL (26). On precontrast $\mathrm{T} 1$ weighted images lesions are usually isointense or hypointense (27-29). Strong homogenous enhancement is often present in PCNSL. Some cases $(0 \%$ to $13 \%)$ show ring enhancement, and a few cases show open-ring enhancement and a "notch sign", which is a deep, abnormal depression at the tumor margin (30). Rare cases $(0 \%$ to $1 \%)$ without enhancement have been described $(31,32)$ usually in the setting of low-grade disease (33) or intravascular lymphomatosis (34) in immunocompetent patients. Infiltration of the corpus callosum is commonly regarded as the most characteristic sign of PCNSL. Symmetrical lesions involving the genu or splenium of the corpus callosum are referred to as a "mirror pattern" or "butterfly pattern".
PCNSLs tend to show subependymal and leptomeningeal spread (97\% of cases) $(35,36)$. Linear enhancement along perivascular spaces is highly suggestive of PCNSL (37-39) because PCNSL has an affinity for perivascular extension. After steroids are administered, lesions visualized with MRI can disappear in as little as several hours. Some lesions, called vanishing tumors, can disappear spontaneously. About half of vanishing tumors are PCNSLs (40). The eye is involved in $20 \%$ to $25 \%$ of patients with PCNSL (41). Ocular lymphoma can be diagnosed with cytologic examination of vitreal aspirate or slit-lamp examination. On MRI, ocular lymphoma can manifest as nodular enhancing lesions in the macula or thickening of the uvea (42). On T2-weighted images lesions are usually isointense or hyperintense, but $40 \%$ of lesions on T2-weighted images show hypointensity, which is attributed to high cellular density of the tumor. On fluid-attenuated inversion recovery (FLAIR) imaging, both tumors and areas of edema appear hyperintense (43). A characteristic finding is bilateral symmetrical hyperintensity with subependymal extension. Because diffusion is usually restricted within lymphomas owing to high cellular density, hyperintensity on diffusion-weighted images (DWIs) and hypointensity on apparent diffusion coefficient (ADC) maps are usually seen. On perfusion-weighted images PCNSL shows low cerebral blood volume (CBV) (44). On susceptibility-weighted images, there are no particular or specific characteristics and no microhemorrhages or calcifications, which are frequently seen in high-grade glioma. Proton magnetic resonance spectroscopy (1H-MRS) in PCNSL shows an almost complete loss of n-acetyl aspartate, a decrease in creatine, a great increase in choline and an increase in lactate $(90 \%)$ ). A reported hallmark of PCNSL on MRS is lipid peaks (90\%) much higher than those in glioblastoma (14\%) $(45,46)$ and high choline_creatine ratios $(>3)$. In immunocompetent patients lesions of PCNSL are usually solitary, located in a cerebral hemisphere, thalamus/basal ganglia, corpus callosum, periventricular region and cerebellum. Necrosis, peripheral enhancement, hemorrhages and calcifications are unusual, and other diagnoses should be considered if any of these features are present. Neuroimaging findings can be atypical in patients who are immunodeficient or who have been treated with radiation, antineoplastic agents, or steroids. Moreover, in recent years, atypical neuroimaging features have sometimes been obtained in immunocompetent patients, even before treatment. Furthermore, 
in 5\% of patients with PCNSL, neuroimaging findings are completely normal (47).

\section{Computed tomography}

On computed tomography (CT) lesions of PCNSL are typically ( $86 \%$ to $92 \%$ of cases) isodense to hyperdense (48) with typically homogenous contrast enhancement.

\section{Nuclear imaging}

Whole-body 18-fluorodeoxyglucose positron emission tomography (18FDG-PET) has an increased sensitivity for the detection of systemic DLBCL over conventional CT staging (49), and has an important role in the exclusion of systemic lymphoma at presentation. 18FDG-PET may assist in differentiating PCNSL from other intracranial malignancies where MRI findings are equivocal (50) 18FDG PET-CT in cases of PCNSL reveals hypermetabolic lesions with increased uptake of FDG $(86 \%)$ as is seen in malignant gliomas. After effective chemotherapy, FDG uptake disappears, and thus, FDG-PET can be used to evaluate the early therapeutic response. After steroid treatment, the degree of hypermetabolic activity in PCNSL may decrease. Nuclear imaging findings facilitate differential diagnosis from other brain tumors and infectious diseases (51). N-isopropyl (123I)-p-iodoamphetamine (IMP) single photon emission CT (SPECT) shows retention in delayed images. (52) On delayed SPECT images the IMP index is 7 in cases of PCNSL but is less than 1 in cases of malignant glioma. High IMP index of PCNSL is similar to that of malignant melanoma. (53) With 201TlClSPECT, homogenously enhancing abnormalities are usually observed. (54) With 67Ga scintigraphy, accumulation is observed 72 hours after injection. The sensitivity of $67 \mathrm{Ga}$ scintigraphy is reported to be $83.1 \%$. (55) On $11 \mathrm{C}$-methionine-PET, uptake is extremely high. The area of increased uptake is often larger than the enhancing lesion on MRI. The area and degree of methionine accumulation in the tumor tissue decrease after radiation therapy. In these nuclear images, immunodeficient patients with PCNSL show characteristics similar to those of immunocompetent patients. (56)

\section{Histology}

Histological confirmation, despite small tissue stereotactic biopsies, establish the diagnosis because, as autopsy studies reveal, most primary CNS lymphomas (PCNSL) extensively infiltrate the brain (32). The majority of PCNSL are diagnosed via stereotactic biopsy or, less commonly, by flow cytometric analysis of cerebrospinal fluid (CSF) lymphocytes. The conventional approach has been to avoid surgical resection given the risk of neurological sequelae and lack of therapeutic benefit (57). However, a recent unplanned secondary analysis of the G-PCNSL-SG-1 trial has challenged this view, describing an apparently superiorprogression-free survival (PFS) for those undergoing complete or subtotal resection (58). However, this study had a number of limitations, and independent verification in a well-designed and controlled study would be required to change practice (59) Rubenstein et al. recently evaluated the utility of CXCL13 (a mediator of B-cell migration) and IL-10 as potential CSF diagnostic biomarkers with the ability to discriminate CNS lymphoma from other CNS. The positive predictive value of CXCL13 and IL10 elevation in CSF was $95 \%$ in the identification of newly-diagnosed HIV-negative PCNSL, with an $88 \%$ negative predictive value. (60)

Primary CNS lymphoma and secondary CNS involvement by systemic DLBCL cannot be distinguished simply on the basis of their morphology and immunophenotype, although CD10 expression, particularly in association with a relatively low Ki67 labeling index $(<50 \%)$, should prompt an intense search of primary extracerebral DLBCL or follicular lymphoma (22). Neuropathological studies $(32,61)$ revealed no correlation of autopsy findings with neuroimaging assessment of tumor burden. MRI underestimates the tumor burden of PCNSL. Bulky disease is seen as a contrast-enhancing lesion because of disruption of the bloodbrain barrier, but microscopic tumor infiltration may lead to T2 hyperintensity or be completely normal, so the disease may infiltrate the entire brain without a corresponding imaging on MRI.

Recently, an algorithm based on a panel of immunohistochemical (IHC) markers (CD10, BCL-6 and MUM-1) was developed to classify DLBCL into two major subtypes: germinal center B-celllike (GCB) (positive for either CD10 or BCL-6 while negative for MUM-1) and activated B-celllike (ABC) (positive for MUM-1 and negative for CD10) (7-10). Three studies have shown that in the CNS, GCB DLBCL might have a better prognosis than ABC DLBCL (62-64).

In contrast to systemic DLBCL, high expression of BCL-2, BCL-6, and MYC by immunohistochemistry is frequent in PCNSL $(70 \%$ of cases studied) (65) and it has been speculated that this may contribute to the adverse prognosis of PCNSL. Recently, the only multi-centre trial to prospectively evaluate PCNSL biomarkers demonstrated that 
BCL-6 expression, but not MYC, correlated with inferior survival (66). Whilst some studies support this finding $(67,68)$, other retrospective analyses found that BCL-6 overexpression correlated with superior outcomes. $(69,70)$

\section{TREATMENT}

\section{Chemotherapy}

High-dose methotrexate (MTX) combined with high-dose cytarabine (AraC) is currently regarded standard treatment (71). There is general consensus that MTX should be administered as a rapid infusion (2-4 hours) at a dose of at least $3 \mathrm{mg} / \mathrm{m}^{2}$ to maximise therapeutic CSF concentrations, at an interval of 14-21 days (72). Modern protocols typically employ between four and eight cycles of HDMTX-based therapy but comparative data on treatment duration is lacking. In the randomised, phase II, multicentre IELSG- 20 trial, $75 \%$ of maximum responses were achieved following the first two cycles of treatment. However, for patients achieving a partial response, further tumor response was observed in patients receiving combination chemotherapy (HD-MTX/Ara-C, 10/18 patients) but not HD-MTX monotherapy (71). In a recent trial of rituximab, HD-MTX, procarbazine and vincristine (R-MPV), complete response rates improved from $47 \%$ after five cycles of chemotherapy to $79 \%$ after seven (73) The addition of thiotepa to HD-MTX and cytarabine was piloted in a small multicentre study $(n=20)$, with inferior results compared to the IELSG20 trial, attributed to a $50 \%$ protocol reduction in cytarabine dose $\left(1 \mathrm{~g} / \mathrm{m}^{2}\right.$ for four doses) (74), although the optimal thiotepa dose in this setting has not been ascertained. The role of thiotepa is currently being evaluated in the ongoing, randomized IELSG32 study (EudraCT number 2009-012432-32).

OSHO-phase II study evaluated patients with newly diagnosed primary central nervous system lymphoma treated with autologous peripheral blood stem-cell transplantation (aPBSCT) and response-adapted whole-brain radiotherapy (WBRT). 23 patients received high-dose methotrexate. In case of at least partial remission, high-dose busulfan/thiotepa (HD-BuTT) followed by aPBSCT was carried out. Patients refractory to induction or without complete remission after HD-BuTT received WBRT. Follow-up shows an overall survival of $35 \%$. In six of seven patients where WBRT could be avoided, no long-term neurotoxicity has been observed and all patients have an excellent quality of life. (75)
Rubenstein et al. used methotrexate, temozolomide, and rituximab (MT-R) induction followed by etoposide and cytarabine (EA) consolidation in a prospective multi-centre trial of 44 patients, achieving a two-year PFS of $57 \%$ and four-year OS of $65 \%(76)$

Ferreri et al, in a phase II trial assessed first-line MATILDE chemotherapy and response-tailored radiotherapy in 41 HIV negative patients with PCNSL. Overall response rate was $76 \%$ after chemotherapy and $83 \%$ after chemotherapy \pm radiotherapy, with a 5 -year overall survival of $30 \% \pm$ $7 \%$. At 10 years from diagnosis, no patient showed chronic hematologic and nonhematologic toxicities, with a Mini-Mental State Examination score of $\geq 29$ in all cases but one. (77)

\section{Rituximab}

In contrast to the survival advantage witnessed in systemic DLBCL, the benefit of combining rituximab with chemotherapy for PCNSL remains unclear. Rituximab has limited CNS penetration with intravenous administration. Single-agent efficacy was demonstrated in 12 patients with refractory/relapsed PCNSL with radiographic response to intravenous rituximab

monotherapy in 36\% (78) and encouraging response rates have been achieved with rituximab $375-500 \mathrm{mg} / \mathrm{m}^{2}$ in conjunction with combination chemotherapy in single-arm trials $(77,79)$ Although the quality of responses appears to be improved with the addition of rituximab, no survival advan tage has yet been demonstrated on multivariate analysis $(80,81)$. Results from ongoing randomised studies (IELSG32: NCT01011920, and HOVON 105 PCNSL/ALLG NHL24 trial: EudraCT 2009014722-42) are essential to define the role of rituximab in induction therapy for PCNSL.

\section{Whole brain radiation therapy (WBRT)}

It has been suggested that whole brain consolidation radiotherapy has no additional benefit regarding overall survival (OS) after high-dose MTX alone or in combination with ifosfamide (82). Nevertheless, ongoing trials compare whole brain radiotherapy with high-dose chemotherapy followed by autologous stem cell transplantation (auto-SCT) as consolidation (NCT01011920, NCT00863460). Beside whole brain radiotherapy, the application of high-dose chemotherapy with carmustine (BCNU) and thiotepa followed by auto-SCT has been shown to be feasible and highly effective in newly diagnosed eligible patients, but also in the salvage situ- 
ation (83-85). In an attempt to ameliorate the longterm neurocognitive sequelae of WBRT at standard doses, investigators have assessed the value of reduced dose WBRT (rdWBRT). Inferior outcomes have been described with a reduced consolidation WBRT dose (30.6Gy) following CHOD/BVAM induction therapy in a non-randomised comparison (86). Morris et al. recently reported encouraging rates of disease control using $23.4 \mathrm{~Gy}$ radiotherapy as consolidation therapy following the R-MPV protocol, with a PFS of 7.7 years for the selected subgroup $(n=31)$ achieving CR with immunochemotherapy. Prospective neuropsychological evaluation demonstrated no overall cognitive decline, in 12 patients assessed 48 months after rdWBRT. (73)

\section{PROGNOSIS}

Prognosis is poor with a median survival of 17 to 45 months in immunocompetent patients, and

\section{REFERENCES}

1. Dolecek T.A., Propp J.M., Stroup N.E., et al. CBTRUS statistical report: Primary brain and central nervous system tumors diagnosed in the United States in 2005-2009. Neuro-oncol 2012; 14(Suppl 5):v1-49

2. Giannini C., Dogan A., Salomăo D.R. CNS lymphoma: a practical diagnostic approach. J Neuropathol Exp Neurol. 2014 Jun; 73(6):478-94

3. Deckert M., Montesinos-Rongen M., Brunn A., et al. Systems biology of primary CNS lymphoma: From genetic aberrations to modeling in mice. Acta Neuropathol 2014; 127:175-88

4. Kluin P.C., Deckert M., Ferry J.A. Primary diffuse large B-cell lymphoma of the CNS. In: Swerdlow S., Campo E., Harris N.L., et al, eds. WHO Classification of Tumours of Haematopoietic and Lymphoid Tissue (IARC WHO Classification of Tumours). Lyon, France: IARC, 2008; 240-41

5. Shiels M.S., Pfeiffer R.M., Hall H.I., et al. Proportions of Kaposi sarcoma, selected non-Hodgkin lymphomas, and cervical cancer in the United States occurring inpersons with AIDS, 1980-2007. JAMA 2011; 305:1450-9

6. Montesinos-Rongen M., Brunn A., Bentink S., et al. Gene expression profiling suggests primary central nervous system lymphomas to be derived from a late germinal center $B$ cell. Leukemia. 2008; 22:400-405.

7. Tun H.W., Personett D., Baskerville K.A., et al. Pathway analysis of primary central nervous system lymphoma. Blood. 2008; 111:3200-3210.

8. Sung C.O., Kim S.C., Karnan S., et al. Genomic profiling combined with gene expression profiling in primary central nervous system lymphoma. Blood. 2011; 117:1291-1300.

9. Rubenstein J.L., Fridlyand J., Shen A., et al. Gene expression and angiotropism in primary CNS lymphoma. Blood. 2006; 107:3716-3723

10. Schwindt H., Vater I., Kreuz M., et al. Chromosomal imbalances and partial uniparental disomies in primary central nervous system lymphoma. Leukemia. 2009; 23:1875-1884.

11. Braggio E., McPhail E.R., Macon W., et al. Primary central nervous system lymphomas: a validation study of array-based comparative only $20-30 \%$ of cases can be cured successfully (17). Two international prognostic scores have been developed to predict outcome in PCNSL: (1) the International Extranodal Lymphoma Study Group (IELSG) score, which distinguishes three prognostic groups based on serum lactate dehydrogenase (LDH), age, Eastern Cooperative Oncology Group (ECOG) performance status, involvement of Deep Brain Structures (periventricular regions, basal ganglia, brainstem, and/or cerebellum), and cerebrospinal fluid (CSF) protein concentration (87) and (2) the Memorial Sloan Kettering Cancer Center (MSKCC) score, which also distinguishes three groups but only according to age and Karnofsky Performance Status (KPS). (88)

\section{Acknowledgements}

Author thank Mrs. Monica Marinescu for the proofreading of the English version of the manuscript. genomic hybridization in formalin-fixed paraffin-embedded tumor specimens. Clin Cancer Res. 2011;17:4245-4253.

12. Gonzalez-Aguilar A., Idbaih A., Boisselier B., et al. Recurrent mutations of MYD88 and TBL1XR1 in primary central nervous system lymphomas. Clin Cancer Res. 2012; 18:5203-5211.

13. Morin R.D., Mendez-Lago M., Mungall A.J., et al. Frequent mutation of histone-modifying genes in non-Hodgkin lymphoma. 2011; 476:298-303.

14. Parsons D.W., Jones S., Zhang X., et al. An integrated genomic analysis of human glioblastoma multiforme. Science. 2008; 321:1807-1812.

15. Bruno A., Boisselier B., Labreche K., et al. Mutational analysis of primary central nervous system lymphoma. Oncotarget. $2014 \mathrm{Jul} 15$; 5(13):5065-75.

16. Sierra del Rio M., Rousseau A., Soussain C., et al. Primary CNS lymphoma in immunocompetent patients. Oncologist 2009; 14:526-39.

17. Aki H., Uzunaslan D., Saygin C., et al. Primary central nervous system lymphoma in immunocompetent individuals: a single center experience. Int J Clin Exp Pathol 2013; 6(6):1068-1075

18. Namekawa M. Malignant lymphoma in the central nervous system: overview. (Article in Japanese) Brain Nerve. 2014 Aug; 66(8):907-16.

19. Bataille B., Delwail V., Menet E., et al. Primary intracerebral malignant lymphoma: report of 248 cases. J Neurosurg. 2000; 92(2):261-266

20. Gerstner E.R., Batchelor T.T. Primary central nervous system lymphoma. Arch Neurol 2010; 67:291-7

21. Abrey L.E., Batchelor T.T., Ferreri A.J., et al. International Primary CNS Lymphoma Collaborative Group. Report of an international workshop to standardize baseline evaluation and response criteria for primary CNS lymphoma. J Clin Oncol. 2005; 23(22):5034-5043.

22. Deckert M., Brunn A., Montesinos-Rongen M., et al. Primary lymphoma of the central nervous system-a diagnostic challenge (published online ahead of print August 16, 2013). Hematol Oncol.

23. Coulon A., Lafitte F., Hoang-Xuan K., et al. Radiographic findings in 37 cases of primary CNS lymphoma in immunocompetent patients. Eur Radiol 2002; 12:329-340. 
24. Küker W., Nägele T., Korfel A., et al. Primary central nervous system lymphomas (PCNSL): MRI features at presentation in 100 patients. J Neurooncol 2005; 72:169-177

25. Gliemroth J., Kehler U., Gaebel C., et al. Neuroradiological findings in primay cerebral lymphoma of non-AIDS patients. Clin Neurol Neurosurg 2003; 105:78-86.

26. Jahnke K., Korfel A., O'Neill B.P., et al. International study on low-grade primary central nervous system lymphoma. Ann Neurol 2006; 59:755-762

27. Hochberg F.H., Baehring J.M., Hochberg E.P. Primary CNS lymphoma: diagnosis. Nat Clin Pract Neurol 2007; 3:24-35.

28. Haldorsen I.S., Espeland A., Larsson E.M. Central nervous system lymphoma: characteristic findings on traditional and advanced imaging. AJNR Am J Neuroradiol 2011; 32:984-992.

29. Roman-Goldstein S.M., Goldman D.L., Howieson J., et al. MR of primary CNS lymphoma in immunologically normal patients. AJNR Am J Neuroradiol 1992; 13:1207-1213.

30. Zhang D., Hu L.B., Henning T.D., et al. MRI findings of primary CNS lymphoma in 26 immunocompetent patients. Korean J Radiol 2010; 11:269-277.

31. Küker W., Nägele T., Korfel A., et al. Primary central nervous system lymphomas (PCNSL): MRI features at presentation in 100 patients. J Neurooncol 2005; 72:169-177.

32. Lai R., Rosenblum M.K., DeAngelis L.M. Primary CNS lymphoma: A whole-brain disease? Neurology 2002; 59:1557-1562

33. Jahnke K., Korfel A., O'Neill B.P., et al. International study on low-grade primary central nervous system lymphoma. Ann Neurol 2006; 59:755-762

34. Williams R.L., Meltzer C.C., Smirniotopoulos J.G., et al. Cerebral MR imaging in intravascular lymphomatosis. AJNR Am J Neuroradiol 1998; 19:427-431.

35. Gliemroth J., Kehler U., Gaebel C., et al. Neuroradiological findings in primay cerebral lymphoma of non-AIDS patients. Clin Neurol Neurosurg 2003; 105:78-86.

36. Bühring U., Herrlinger U., Krings T., et al. MRI features of primary central nervous system lymphomas at presentation. Neurology 2001; 57:393-396.

37. Go J.L., Lee S.C., Kim P.E. Imaging of primary central nervous system lymphoma. Neurosurg Focus 2006; 21:E4

38. Eichler A.F., Batchelor T.T. Primary central nervous system lymphoma: presentation, diagnosis and staging. Neurosurg Focus 2006; 21:E15

39. Atlas S.W. Magnetic resonance imaging of the brain and spine, ed 3. 2002; Lippincott Williams \& Wilkins, Philadelphia.

40. Bromberg J.E.C., Siemers M.D., Taphoorn J.B. Is a "vanishing tumor" always a lymphoma? Neurology 2002; 59:762-764.

41. Gunduz K., Pulido J.S., McCannel C.A, et al. Ocular manifestations and treatment of central nervous system lymphomas. Neurosurg Focus 2006; 21:E9

42. Küker W., Herrlinger U., Grönewäller E., et al. Ocular manifestation of primary nervous system lymphoma: what can be expected from imaging? J Neurol 2002; 249:1713-1716.

43. Hochberg F.H., Baehring J.M., Hochberg E.P. Primary CNS lymphoma: diagnosis. Nat Clin Pract Neurol 2007; 3:24-35.

44. Calli C., Kitis O., Yunten N., et al. Perfusion and diffusion MR imaging in enhancing malignant cerebral tumors. Eur J Radiol 2006; 58:394-403.

45. Zacharia T.T., Law M., Naidich T.P., et al. Central nervous system lymphoma characterization by diffusion-weighted imaging and MR spectroscopy. J Neuroimaging 2008; 18:411-417.

46. Raizer J.J., Koutcher J.A., Abrey L.E., et al. Proton magnetic resonance spectroscopy in immunocompetent patients with primary central nervous system lymphoma. J Neurooncol 2005; 71:173-180.

47. Thaler P.B., Yi Li J., Isakov Y., et al. Normal or non-diagnostic neuroimaging studies prior to the detection of malignant primary brain tumors. J Clin Neurosci 2012; 19:411-414.

48. Haldorsen I.S., Espeland A., Larsson E.M. Central nervous system lymphoma: characteristic findings on traditional and advanced imaging. AJNR Am J Neuroradiol 2011; 32:984-992.
49. Mohile N.A., Deangelis L.M., Abrey L.E. The utility of body FDG PET in staging primary central nervous system lymphoma. Neuro Oncol. 2008; 10:223-8.

50. Makino K., Hirai T., Nakamura H., et al. Does adding FDG-PET to MRI improve the differentiation between primary cer ebral lymphoma and glioblastoma? Observer performance study. Ann Nucl Med. $2011 ; 25: 432-438$

51. Adachi K., Yamaguchi F., Node Y., et al. Neuroimaging of Primary Central Nervous System Lymphoma in Immunocompetent Patients: Comparison of Recent and Previous Findings. J Nippon Med Sch 2013; 80:174-183

52. Shinoda J., Yano H., Murase S., et al. High 123I-IMP retention on SPECT image in primary central nervous system lymphoma. J Neurooncol 2003; 61:261-265

53. Abe K., Sasaki M., Koga H., et al. Clinical role of 123I- IMP SPECT for the differential diagnosis of ocular malignant melanoma: time-course analysis. Nucl Med Commun 2007; 28: 567-573.

54. Kasamon Y.L., Ambinder R.F. AIDS-related primary central nervous system lymphoma. Hematol Oncol Clin North Am 2005; 19:665-687.

55. Nishiyama Y., Yamamoto Y., Toyama Y., et al. Usefulness of $67 \mathrm{Ga}$ scintigraphy in extranodal malignant lymphoma patients. An Nucl Med 2003; 17:657-662.

56. Ogawa T., Kanno I., Hatazawa J., et al. Methionine PET for follow-up of radiation therapy of primary lymphoma of the brain. RadioGraphics 1994; 14:101-110.

57. Bellinzona M., Roser F., Ostertag H., et al. Surgical removal of primary central nervous system lymphomas (PCNSL) presenting as space occupying lesions: a series of 33 cases. Eur J Surg Oncol. 2005; 31:100-5.

58. Weller M., Martus P., Roth P., Thiel E., Korfel A. Surgery for primary CNS Iymphoma? Challenging a paradigm. Neuro Oncol. 2012; 14:1481-4.

59. Phillips E.H., Fox C.P., Cwynarski K. Primary CNS lymphoma. Curr Hematol Malig Rep. 2014 Sep; 9(3):243-53.

60. Rubenstein J.L., Wong V.S., Kadoch C., et al. CXCL13 plus interleukin 10 is highly specific for the diagnosis of CNS lymphoma. Blood. 2013; 121:4740-8.

61. Koeller K.K., Smirniotopoulos J.G., Jones R.V. Primary central nervous system lymphoma: radiologic-pathologic correlation. Radiographics 1997; 17: 1497-1526.

62. Hans C.P., Weisenburger D.D., Greiner T.C., et al. Confirmation of the molecular classification of diffuse large B-cell lymphoma by immunohistochemistry using a tissue microarray. Blood 2004; 103:275-82.

63. Lin C.H., Kuo K.T., Chuang S.S., et al. Comparison of the expression and prognostic significance of differentiation markers between diffuse large B-cell lymphoma of central nervous system origin and peripheral nodal origin. Clin Cancer Res 2006; 12:1152-6.

64. Bhagavathi S., Sharathkumar A., Hunter S., et al. Activated B-cell immunophenotype might be associated with poor prognosis of primary central nervous system lymphomas. Clin Neuropathol 2008; 27:13-20.

65. Brunn A., Nagel I., Montesinos-Rongen M., et al. Frequent triple-hit expression ofMYC, BCL2, and BCL6 in primary lymphoma of the central nervous system and absence of a favorable MYC(low) BCL2 (low) subgroup may underlie the inferior prognosis as compared to systemic diffuse large B cell lymphom. Acta Neuropathol. 2013; 126:603-5.

66. Rubenstein J.L., Hsi E.D., Johnson J.L., et al. Intensive chemotherapy and immunotherapy in patients with newly diagnosed primary CNS Iymphoma: CALGB 50202 (Alliance 50202). J Clin Oncol. 2013; 31:3061- 8

67. Cady F.M., O'Neill B.P., Law M.E., et al. Del(6)(q22) and BCL6 rearrangements in primary CNS lymphoma are indicators of an aggressive clinical course. J Clin Oncol. 2008; 26:4814-9.

68. Montesinos-Rongen M., Godlewska E., Brunn A., et al. Activating L265P mutations of the MYD88 gene are common in primary central nervous system lymphoma. Acta Neuropathol. 2011; 122:791-2. 
69. Braaten K.M., Betensky R.A., de Leval L., et al. BCL-6 expression predicts improved survival in patients with primary central nervous system lymphoma. Clin Cancer Res. 2003; 9:1063-9.

70. Levy O., DeAngelis L.M., Filippa D.A., et al. Bcl-6 predicts improved prognosis in primary central nervous system lymphoma. Cancer. 2008; 112:151-6.

71. Ferreri A.J., Reni M., Foppoli M., et al. High-dose cytarabine plus high-dose methotrexate versus high-dose methotrexate alone in patients with primary CNS lymphoma: a randomised phase 2 trial. Lancet. 2009; 374 (9700):1512-20.

72. Joerger M., Huitema A.D.R., Illerhaus G., et al. Rational administration schedule for high-dose methotrexate in patients with primary central nervous system lymphoma. Leuk Lymphoma. 2012; 53:1867-75.

73. Morris P.G., Correa D.D., Yahalom J., et al. Rituximab, methotrexate, procarbazine, and vincristine followed by consolidation reduceddose whole-brain radiotherapy and cytarabine in newly diagnosed primary CNS lymphoma: final results and long-term outcome. J Clin Oncol. 2013; 31:3971-9.

74. Ferreri A.J.M., Licata G., Foppoli M., et al. Clinical relevance of the dose of cytarabine in the upfront treatment of primary CNS lymphomas with methotrexate-cytarabine combination. Oncologist. $2011 ; 16: 336-41$

75. Kiefer T., Hirt C., Späth C., et al. Long-term follow-up of high-dose chemotherapy with autologous stem-cell transplantation and response-adapted whole-brain radiotherapy for newly diagnosed primary CNS lymphoma: results of the multicenter Ostdeutsche Studiengruppe Hamatologie und Onkologie OSHO-53 phase II study. Ann Oncol. 2012 Jul; 23(7):1809-12.

76. Rubenstein J.L., Hsi E.D., Johnson J.L., et al. Intensive chemotherapy and immunotherapy in patients with newly diagnosed primary CNS lymphoma: CALGB 50202 (Alliance 50202). J Clin Oncol. 2013; 31:3061-8.

77. Ferreri A.J., Ciceri F., Brandes A.A., et al. MATILDE chemotherapy regimen for primary CNS lymphoma: results at a median follow-up of 12 years. Neurology. 2014 Apr 15; 82(15):1370-3.

78. Batchelor T.T., Grossman S.A., Mikkelsen T., et al. Rituximab monotherapy for patients with recurrent primary CNS lymphoma. Neurology. 2011/03/09 ed. 2011; 76:929-30.
79. Fritsch K., Kasenda B., Hader C., et al. Immunochemotherapy with rituximab, methotrexate, procarbazine, and lomustine for primary CNS lymphoma (PCNSL) in the elderly. Ann Oncol. 2011; 22:2080-5.

80. Birnbaum T., Stadler E.A., Von Baumgarten L, et al. Rituximab significantly improves complete response rate in patients with primary CNS lymphoma. J Neurooncol. 2012; 109:285-91.

81. Gregory G., Arumugaswamy A., Leung T., et al. Rituximab is associated with improved survival for aggressive B cell CNS lymphoma. Neuro Oncol. 2013; 15:1068-73.

82. Thiel E., Korfel A., Martus P., et al. High-dose methotrexate with or without whole brain radiotherapy for primary CNS lymphoma (G-PCNSL-SG-1): a phase 3, randomised, non-inferiority trial. Lancet Oncol. 2010; 11(11):1036-47.

83. Soussain C., Hoang-Xuan K., Taillandier L., et al. Intensive chemotherapy followed by hematopoietic stem-cell rescue for refractory and recurrent primary CNS and intraocular lymphoma: Societe Francaise de Greffe de Moelle Osseuse-Therapie Cellulaire. J Clin Oncol. 2008; 26(15):2512-8.

84. Illerhaus G., Marks R., Ihorst G., et al. High-dose chemotherapy with autologous stem-cell transplantation and hyperfractionated radiotherapy as first-line treatment of primary CNS lymphoma. J Clin Oncol. 2006; 24 (24):3865-70.

85. Illerhaus G., Muller F., Feuerhake F., et al. High-dose chemotherapy and autologous stem-cell transplantation without consolidation radiotherapy as first-line treatment for primary lymphoma of the central nervous system. Haematologica. 2008; 93(1):147-8.

86. Bessell E.M., López-Guillermo A., Villá S., et al. Importance of radiotherapy in the outcome of patients with primary CNS lymphoma: an analysis of the CHOD/BVAM regimen followed by two different radiotherapy treatments. J Clin Oncol. 2002; 20:231-6.

87. Ferreri A.J., Blay J.Y., Reni M., et al. Prognostic scoring system for primary CNS lymphomas: the International Extranodal Lymphoma Study Group experience. J Clin Oncol. 2003; 21(2):266-72.

88. Abrey L.E., Ben-Porat L., Panageas K.S., Yahalom J., Berkey B., Curran W., et al. Primary central nervous system lymphoma: the Memorial Sloan-Kettering Cancer Center prognostic model. J Clin Oncol. 2006; 24(36):5711-5. 